\title{
Negative Einflüsse auf Produktivität und Wohlbefinden von Diabetikern
}

Laut einer Studie von Geelhoed-Duijvestijn und Kollegen sind $11 \%$ der weltweiten Gesundheitsausgaben (465 Mrd.€) auf Diabetes zurückzuführen. Viele Behandler und Menschen mit Diabetes schrecken aus Angst vor Hypoglykämien vor einer Insulintherapie zurück. Bislang existierten kaum länderspezifische Angaben zu der durch Hypoglykämien bedingten Inanspruchnahme von Leistungen der Gesundheitssysteme. Ziel dieser Studie war es daher, den Einfluss von nicht schweren hypoglykämischen Ereignissen auf das Gesundheitssystem und auf das Wohlbefinden der Patienten zu ermitteln. Zudem sollte der Gebrauch von Leistungen der Gesundheitssysteme und der Therapieerfolg nach nicht schweren hypoglykämischen Ereignissen bei Menschen mit Typ-1- und Typ-2-Diabetes ermittelt werden.

\section{Studiendesign}

In 7 europäischen Ländern (Österreich, Dänemark, Finnland, Niederlande, Norwegen, Schweden und Schweiz) wurden Patientendaten mittels einer webbasierten Umfrage erhoben. Voraussetzung war, dass bei den Befragten Diabetes diagnostiziert war, sie mit Insulin behandelt wurden und mindestens 15 Jahre alt waren. Die Studienteilnehmer wurden nach Typ-1- und Typ-2-Diabetes kategorisiert. Bei Menschen mit Typ-2-Diabetes wurde weiterhin nach der jeweiligen Insulintherapie unterschieden:

- $\quad$ BOT - basal-only therapy (reine Basaltherapie),

- BB - basal-bolus therapy (Basis-Bolus-Therapie),

- $\quad$ - other insulin regimens (andere Insulintherapien).

Die Teilnehmer wurden 4 Mal zu den Folgen möglicher nicht schwerer hypoglykämischer Ereignisse in den 7 vorangegangenen Tagen befragt. Dabei wurden die Kontakte zu medizinischem Fachpersonal, die Anzahl zusätzlich verbrauchter Blutzuckerteststreifen und der auf nicht schwere hypoglykämische Ereignisse zurückzuführende Arbeitsausfall erfasst. Außerdem wurden die Teilnehmer hinsichtlich der Einschränkungen

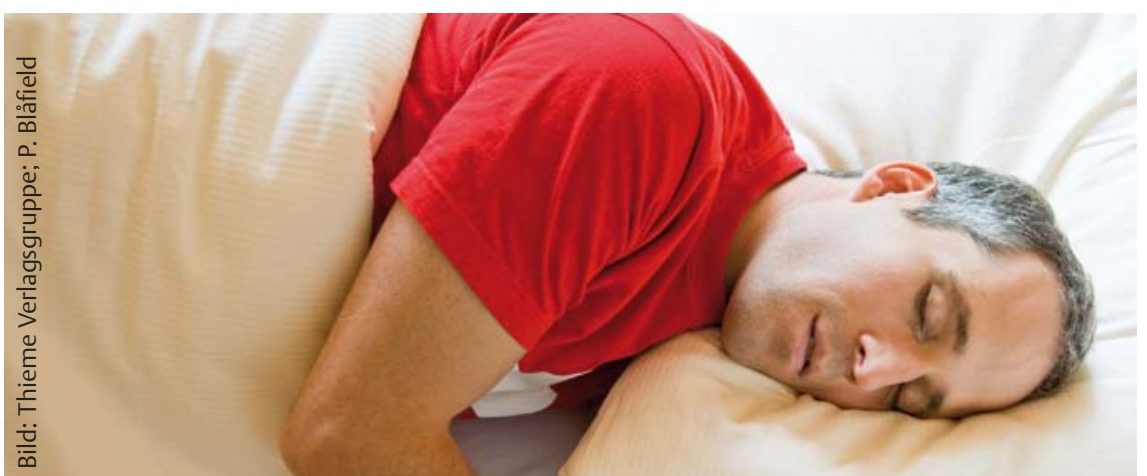

ihres Wohlbefindens durch nicht schwere hypoglykämische Ereignisse befragt.

\section{Ergebnisse}

In die Ergebnisse der Untersuchung flossen die Antworten von 3827 Studienteilnehmern ein. Insgesamt konnten 11440 Wochenberichte ausgewertet werden. Je Woche und Teilnehmer traten im Schnitt 1,8 (Typ-1-Diabetes), 0,4 (Typ-2, BOT), 0,7 (Typ-2, BВ) bzw. 0,5 (Typ-2, O) nicht schwere hypoglykämische Ereignisse auf. Nach einem nicht schweren hypoglykämischen Ereignis wurden in 2,3\% (Typ-1), 8,9\% (Тyp-2, ВОТ), 7,0\% (Тyp-2, ВВ) bzw. 9,7\% (Typ-2, 0) der Fälle medizinisches Fachpersonal kontaktiert. Im Schnitt wurden in der auf ein nicht schweres hypoglykämisches Ereignis folgenden Woche 3 Blutzuckermessstreifen zusätzlich verbraucht (2,9 bei Typ-1, 2,7 bei Typ-2, BOT, 3,1 bei Typ-2, BB und 3,8 bei Typ-2, 0). 13,6\% der Teilnehmer reduzierten nach einem nicht schweren hypoglykämischen Ereignis die Insulindosis.

Die berufstätigen Studienteilnehmer berichteten nach 9,7\% aller nicht schweren hypoglykämischen Ereignisse von einem Arbeitszeitausfall (10,2\% nach am Tag auftretenden Hypoglykämien, 8,1\% nach nächtlichen Hypoglykämien). Der Arbeitszeitausfall betrug bei am Tag auftretenden nicht schweren hypoglykämischen Ereignissen durchschnittlich 84,3 Minuten, bei nächtlichen nicht schweren hypoglykämischen Ereignissen sogar 169,6 Minuten. 10\% der nicht schweren hypoglykämischen Ereignisse führten dazu, dass die Teilnehmer ihren Arbeitstag neu planen mussten. Bei 27\% der Ereignisse klagten die Teilnehmer nach einer Hypoglykämie über Konzentrationsschwierigkeiten. 12\% der Studienteilnehmer hatten das Gefühl, dass ihre Kollegen die Auswirkungen des hypoglykämischen Ereignisses bemerkten.

Die Zeit bis zum Erreichen einer normalen Leistungsfähigkeit war nach nächtlichen nicht schweren hypoglykämischen Ereignissen im Vergleich zu Hypoglykämien am Tag höher (80,5 Minuten vs. 50,4 Minuten). Nicht schwere hypoglykämische Ereignisse gehen häufig mit negativen Auswirkungen auf das Wohlbefinden einher. 58\% der Teilnehmer gaben an, am Tag nach einem nicht schweren hypoglykämischen Ereignis müde oder erschöpft zu sein. $40 \%$ der Teilnehmer klagten über eine verringerte Aufmerksamkeit, 24\% der Teilnehmer über eine erhöhte Reizbarkeit.

\section{Schlussfolgerungen}

Insgesamt führten nächtliche nicht schwere hypoglykämische Ereignisse zu stärkeren negativen Einflüssen auf die Produktivität und das Wohlbefinden der Teilnehmer als am Tag auftretende nicht schwere hypoglykämische Ereignisse. Die von Geelhoed-Duijvestijn und Kollegen durchgeführte Studie zeigt, dass der Prävention insbesondere von nächtlichen Hypoglykämien vor dem Hintergrund der ökonomischen Folgen und des reduzierten Wohlbefindens der Betroffenen eine große Bedeutung zukommt.

sts

Quelle: Geelhoed-Duijvestijn PH, Pedersen-Bjerg $U$, Weitgasser R et al. Effects of patient-reported non-severe hypoglycemia on healthcare resource use, work-time loss, and wellbeing in insulintreated patients with diabetes in seven European countries. J Med Econ 2013; 16: 1453-1461 